INVESTIGACIÓN

Recibido: 12/03/2021 --- Aceptado: 12/03/2021 --- Publicado: 24/05/2021

\title{
AMPLIANDO LOS HORIZONTES DEL NEUROMARKETING: DISMINUCION DE LOS PROBLEMAS ADICTIVOS EN LA COMUNIDAD
}

\section{Widening the horizons of neuromarketing: Decreasing Addictive Problems in the Community}

Javier Cervantes-Aldana. Universidad Nacional Autónoma de México (UNAM). México. jcervantes@fca.unam.mx

Miryam Domínguez-Marín. Universidad Nacional Autónoma de México (UNAM). México. mdgzmarin@outlook.com

Cómo citar el artículo:

Cervantes-Aldana, J. y Domínguez-Marín, M. (2021). Ampliando los horizontes del neuromarketing: disminución de los problemas adictivos en la comunidad. Vivat Academia. Revista de Comunicación, 154, 19-40. http:/ / doi.org/10.15178/va.2021.154.e1322 http://www.vivatacademia.net/index.php/vivat/article/view/1322

\section{RESUMEN}

Esta ponencia con carácter de un ensayo teórico pretende ampliar los horizontes del neuromarketing hacia causas sociales, pero sustentado con la recopilación de algunos ejemplos de estudios prácticos de investigadores del neuromarketing, así como de las causas de las adicciones y su afectación en el cerebro, debido a las substancias adictivas. Las investigaciones han logrado grandes avances en la etiología y dinámica de las adicciones, por lo que se propone en esta ponencia que el neuromarketing puede aplicarse también en las campañas para prevenir las adicciones en la sociedad, principalmente entre adolescentes. Es proponer a la comunidad académica y científica la creación de una nueva rama inexistente actualmente como área específica de investigación a futuro denominada neuromarketing social (o de causas sociales) que combine las investigaciones en mercadotecnia con los hallazgos en las neurociencias. A pesar de este conocimiento sobre la reacción cerebral en las adicciones que han estudiado los neurocientíficos, falta todavía que se conecte este conocimiento de las reacciones adictivas y emocionales con campañas preventivas que puedan ayudar a disminuir estos problemas antes de volverse una adicción. La aplicación de las 
Cervantes-Aldana, J. y Domínguez-Marín, M.

Ampliando los horizontes del neuromarketing: disminución de los problemas adictivos en la comunidad

herramientas de medición de las neurociencias puede mejorar los resultados de las campañas preventivas. En este sentido es un llamado abierto a profesores, alumnos e investigadores de instituciones académicas para realizar más investigaciones prácticas para acumular experiencias nuevas que permitan crecer los casos prácticos de solución de problemas del neuromarketing social.

PALABRAS CLAVE: Neuromarketing - publicidad - adicciones - adolescentes-social -marketing - neurociencias- psicología del consumidor- marketing social

\section{ABSTRACT}

This article created a controversy among marketing professors when they said that marketing should remain in the commercial area of business. Similarly, this paper is a theoretical essay, aimed to broaden the horizons of Neuromarketing towards social causes, but supported by the compilation of some examples of practical studies by Neuromarketing researchers, as well as research on the causes of addictions and their effects in the brain due to addictive substances. The research has made great progress in the etiology and dynamics of addictions, which is why it's proposed in this paper that Neuromarketing can also be applied in campaigns to prevent addictions in society, mainly among adolescents. In this sense, the purpose here is to suggest to the academic and scientific community the creation of a new branch that currently does not exist as a specific area of future research called "Social Neuromarketing" (or of social causes) that combines research in marketing with findings in neurosciences. Despite this knowledge about the brain reaction in addictions that neuroscientists have studied, it's still necessary to connect this knowledge of addictive and emotional reactions with preventive campaigns that can help reduce these problems before becoming an addiction. The application of neuroscience measurement tools can improve the results of preventive campaigns. In this sense, it's an open call to professors, students and researchers from academic institutions to carry out more practical research to accumulate new experiences that allow practical cases to grow up problem solving on social neuromarketing.

KEY WORDS: Neuromarketing - advertising - addictions - teenagers - social marketing - neurosciences- consumer psychology- social marketing

\section{INTRODUCCIÓN}

La mercadotecnia o marketing tiene como propósito la satisfacción de necesidades de los consumidores (Stanton, 2000; McCarthy y Perreeault, 1997; Fischer de la Vega y Espejo; Callado, 2004; Mercado, 1998). En general, el marketing ha estudiado la conducta del consumidor, sus necesidades y deseos, la segmentación de los mercados, los factores ambientales, y se concentra en la creación de estrategias para productos, servicios, promoción, precios, distribución (Kerin y et al., 2009). 

comunidad

Desde nuestro punto de vista, el marketing se creó como una disciplina "eclética" pues muchos de sus conocimientos teóricos se han basado en técnicas y conocimientos de otras disciplinas, principalmente sociales, como la economía, antropología, sociología o la psicología (Münich Galindo y García-Martínez, 1990), pero también han utilizado la estadística, las matemáticas y más recientemente el uso de las herramientas neurocientíficas, que brindan información objetiva acerca de las preferencias y gustos de los consumidores.

Sin embargo, se ha pensado que el marketing no sólo puede influenciar la toma de decisiones de compra, sino también el cambio de ideas y conductas sociales (Kotler y Roberto, 1992). Kotler y Zaltman en 1971 publican el artículo "Social Marketing: An Approach to Planned Change" sentando así las bases del marketing social. Este enfoque del marketing centrado en problemas sociales generó el interés de diversas campañas como son, la de prevención de cáncer de mama, la alimentación de bebés con leche materna, la importancia de la vacunación o la prevención contra las adicciones, entre otros temas.

\section{OBJETIVOS}

El objetivo de esta investigación es explorar de qué manera el conocimiento de las neurociencias aplicadas al marketing puede apoyar en el desarrollo de estrategias de comunicación social para auxiliar en la prevención de las adicciones. Además de proponer una nueva área de estudio que llamaremos neuromarketing social y plantear a los investigadores y académicos el desarrollo de estudios prácticos sobre problemáticas sociales que contribuyan al bienestar de la comunidad.

\section{METODOLOGÍA}

En la primera etapa, este artículo pretende ser un ensayo teórico mediante revisión bibliográfica, ya que existe muy poca evidencia sobre la aplicación del neuromarketing con causas sociales. Partimos de una nueva área que se desea proponer desde el punto de vista académico de la que se carece evidencia científica de su existencia, a fin de que se desarrollen estudios y casos prácticos que ayuden a comprobar nuestra propuesta y que resuelvan problemas graves dentro de la sociedad como es el caso de la adicción a las drogas lícitas como el alcohol y la nicotina, así como a las ilícitas como la marihuana, la cocaína, las anfetaminas y los opiáceos, por mencionar los más importantes; además de prevenir la adicción a los videojuegos, al internet y la ludopatía.

Nos basaremos en la revisión de la literatura del marketing, del neuromarketing y del marketing social en particular que conformará nuestro sustento teórico, así como de los estudios de neuromarketing aplicados a conductas adictivas. Agregaremos ejemplos de casos prácticos en investigaciones del neuromarketing y las neurociencias en general, que permitan elaborar una propuesta de estrategia de comunicación contra la drogadicción en particular pero que pueda ser aplicable a otras conductas adictivas. 
En una segunda etapa (no contemplada aquí), proponemos llevar a cabo experimentos de corte científico que validen los supuestos de que el neuromarketing social debe ser considerado como un área futura de estudio, que puede aplicarse con éxito a coadyuvar en la prevención de problemas de adicción.

\section{Antecedentes del marketing social}

En 1969, Kotler y Levy, publicaron el artículo Broadening the Concept of Marketing (Ampliando el concepto de marketing), creando un gran debate entre los académicos de esta disciplina, estableciendo una nueva era de las aplicaciones a las organizaciones no comerciales (Cervantes Aldana, 1980).

Las investigaciones para aplicar el marketing con fines sociales se incrementaron desde entonces y a partir de 1971, cuando Kotler y Zaltman publicaron el artículo Social Marketing: An Approach to Planned Change y se dio a conocer el marketing social, aunque hay que reconocer que inducir cambios en la sociedad se ha intentado desde tiempo atrás pero no se le identificaban estos esfuerzos con el marketing social.

Hay muchas definiciones sobre mercadotecnia social, pero fueron Kotler y Zaltman (1971) quienes dieron la primera definición de marketing social: “Mercadotecnia social es el diseño, implementación y control de programas calculados que puedan influir en la aceptabilidad de ideas sociales e involucrando consideraciones de planeación de producto, precio, comunicación, distribución e investigación de mercado" (1971, p.5).

Las definiciones de mercadotecnia social se han centrado en el concepto de modificar el comportamiento para lograr cambios voluntarios de las personas y que de esta forma se beneficien tanto el individuo como la sociedad en general (Armario, 1997; Andersen, 1994). Otra definición más reciente de Kotler y Lee (2008) donde además de considerar los conceptos de influir en el comportamiento para beneficio de la sociedad, incorporan el término de "creación de valor" que se ha vuelto común dentro de las definiciones contemporáneas de la mercadotecnia. Estos autores definieron la mercadotecnia social como: "el proceso de aplicar principios y técnicas de mercadotecnia para crear, comunicar y entregar valor para influir el comportamiento del público objetivo para beneficio de la sociedad (salud pública, seguridad, medio ambiente y comunidades) así como a la audiencia objetivo" (Kotler y Lee (2008, p. 7). En esta definición vemos cómo la mercadotecnia social puede servir a diferentes causas que necesitan ser resueltas como el cuidado del medio ambiente o los asuntos que preocupan a la salud pública, incluyendo desde luego el caso de las adicciones que nos ocupa en este artículo.

El exceso del consumo de productos anunciados en los medios de comunicación masiva como los licores, los cigarros y los alimentos de alta densidad energética, han sido relacionados con distintas problemáticas sociales, como el alcoholismo (Smith y Foxcroft, 2009), el tabaquismo (Pierce y Gilpin, 2005) y la obesidad (Witkowski, 2007), que se han convertido en problemas urgentes de salud pública a nivel mundial (Domínguez-Marín, 2017). En tiempos recientes, las campañas de cambio social se

Vivat Academia. Revista de Comunicación. 2021, nº 154, 19-40 
centran en diversos temas como reformas sanitarias (antitabaquismo, prevención contra el abuso de las drogas, alcoholismo, nutrición y buena condición física), reformas para la mejora del medio ambiente (prevención de la contaminación del agua, reducción de emisión de $\mathrm{CO} 2$, protección de refugios para la vida silvestre, protección de especies en peligro de extinción) en reformas educativas (para incrementar la alfabetización de adultos, mejorar las escuelas públicas, mejorar el rendimiento escolar de los estudiantes y profesores) y reformas económicas (para revitalizar ciudades industriales antiguas; fomentar las habilidades y el entrenamiento en el trabajo y atraer a inversionistas extranjeros; (Kotler y Roberto, 1992). Una investigación interesante sobre mercadotecnia social fue la realizada en México para prevenir las enfermedades gastrointestinales en niños menores de 5 años y disminuir su mortalidad mediante sus campañas de salud (Cervantes-Aldana 1980).

En nuestra búsqueda de la literatura, no se ha encontrado un concepto que defina el enfoque social del neuromarketing, porque la gran mayoría de los artículos sobre neuromarketing que se han realizado hasta la fecha, han estado enfocados a conocer el impacto de las estrategias de marketing en el cerebro del consumidor, ya sea de la publicidad, la promoción, el diseño de productos, etc., pero todos estos estudios van enfocados al ámbito comercial, buscando incidir en las preferencias de los consumidores. Hasta ahora existen muy pocos estudios académicos que hayan utilizado técnicas neurocientíficas para indagar causas sociales, en un apartado más adelante describiremos estos estudios y analizaremos su importancia.

Estamos seguros de que el trabajo multidisciplinario de ambas áreas (neurociencias y marketing) darán como resultado una nueva línea de investigación que se propone en este artículo denominar "neuromarketing social" y que brindará un conocimiento más profundo y especializado acerca de la influencia de la mercadotecnia lucrativa sobre la dinámica social que genera problemas en la comunidad. El neuromarketing social puede proveer información relevante que coadyuve a resolver este y otros problemas provocados o acrecentados por la mercadotecnia lucrativa (DomínguezMarín, 2017).

Por ello, se han tomado los conceptos de neuromarketing y marketing social para delimitar el concepto, así proponemos la siguiente definición: neuromarketing social es toda investigación en la que son utilizadas técnicas y métodos de las neurociencias en los grupos que interesa influir en la conducta, con el fin de realizar estrategias de marketing social enfocadas en el apoyo de causas, ideas, productos o conductas sociales. En el caso de este artículo, queremos enfocarnos al campo de la prevención de las adicciones y no en su cura.

\section{El problema social de las adicciones}

El abuso de las drogas es un problema social y de salud que afecta la calidad de vida de las personas en el ámbito psicológico, físico y social (Fadei y et al., 2020). Una sustancia de abuso es aquella que comúnmente se utiliza con la finalidad de sentir placer y promueve la dependencia de su uso (Prospero-García et al., 2014). De acuerdo

Vivat Academia. Revista de Comunicación. 2021, nº 154, 19-40 

comunidad

con el Informe Mundial sobre las Drogas (UNODC, 2020), en 2018, alrededor de 269 millones de personas (5\% de la población mundial) entre 15-64 años consumieron algún tipo de droga ilícita, de los cuales 36 millones presentan dependencia y durante el periodo de 2009 a 2018 hubo un aumento del 30\% de la población que las consume. Mientras los países en desarrollo reportaron los mayores índices de crecimiento en el consumo de sustancias de abuso, siendo los adolescentes y los adultos jóvenes los más afectados con un aumento del 16\% durante el periodo del 2000 al 2018.

Según la Organización Mundial de la Salud (OMS, 2004) es una enfermedad física y psicoemocional que provoca la búsqueda y abuso de la droga y se caracteriza por un conjunto de signos y síntomas, es una enfermedad progresiva que puede llevar a la muerte. El Dr. Óscar Prospero-García (2018) neurocientífico especialista en adicciones describe la dependencia a una droga de abuso como la incapacidad de una persona para realizar las actividades diarias cuando carece de la droga, "Una persona adicta utiliza la mayor parte de su tiempo en buscar la droga de la cual es dependiente y en reponerse de sus efectos, así mismo, el sujeto continúa administrándose la sustancia a pesar de que le provoque efectos adversos físicos, sociales y familiares" (ProsperoGarcía, 2018; p.4).

Para conseguir una reducción de la demanda del consumo de drogas es fundamental el desarrollo de medidas preventivas, por ser el instrumento más efectivo en lugar de invertir más dinero en el tratamiento de las propias adicciones. Se sabe que los programas de prevención son más rentables en comparación con los esfuerzos que realizan los gobiernos por curar la adicción (Fadaei y et al., 2020).

\section{Los adolescentes y las drogas de abuso}

Diferentes estudios han mostrado que el inicio del consumo de las drogas se da durante la adolescencia, alrededor de los 16 años y permanece en la edad adulta (Fadaei , et al., 2020). Sin embargo, la edad de experimentación en los últimos años ha disminuido y ahora se presenta entre los 12 y 13 años (Reséndiz-Escobar y et al.,2018; Martínez-Martínez, 2008; Young, et al., 2002). La adolescencia es una etapa difícil en la que se presentan cambios emocionales, sociales y cognitivos, así mismo, las funciones ejecutivas de control de la conducta ejecutadas por la corteza prefrontal, aún se encuentran en desarrollo, podríamos decir que los adolescentes son vulnerables al presentar respuestas impulsivas y conductas de riesgo, como el consumo de alcohol, nicotina y drogas ilícitas (Jadhav y Boutrel, 2019).

Algunos estudios realizados en población adolescente han encontrado que el consumo de sustancias ilícitas aumenta con la edad, la escolaridad y la disponibilidad de dinero para poder comprar la droga (Burrone, et al., 2010). Los adolescentes experimentan por curiosidad, ocio o presión de los pares (Gómez y Muñoz, 2000), este primer consumo puede convertirse en un consumo casual de fines de semana o fiestas donde se presenta la oportunidad, en estos dos tipos de consumo, el experimental o el ocasional, se puede tener éxito con campañas de prevención, sin embargo, cuando el sujeto comienza abusar de las drogas, será difícil, tratar de persuadirlo a través de

Vivat Academia. Revista de Comunicación. 2021, nº 154, 19-40 
Cervantes-Aldana, J. y Domínguez-Marín, M.

Ampliando los horizontes del neuromarketing: disminución de los problemas adictivos en la comunidad

estrategias de marketing social, es por eso que la presente investigación propone el uso del neuromarketing social en la población adolescente no adicta.

Neurobiología de las adicciones

Sistema de motivación y recompensa

El sistema de motivación y recompensa (SMR) o de reforzamiento, es un sistema cerebral que nos permite sentir placer, formado por el área tegmental ventral (ATV) que se activa al llevar a cabo conductas como comer, beber o tener sexo (reforzadores necesarios), generando la liberación de un neurotransmisor llamado dopamina que proyecta al núcleo accumbens donde aumenta su liberación y genera la sensación subjetiva de placer para asegura la repetición de conductas que son críticas para la supervivencia humana (Prospero-García, 2014). A su vez, este sistema es usurpado por las sustancias de abuso (reforzadores vacíos) que, a diferencia de los reforzadores necesarios, sobre activan el ATV generando una liberación masiva de dopamina que produce una sensación subjetiva de placer más intensa. Sin embargo, las sustancias de abuso causan que el sistema de motivación y recompensa reduzca una sobre excitabilidad, por ello, es necesario el aumento de la dosis de droga para conseguir los mismos efectos (Méndez-Díaz, et al., 2017).

\section{Sistema de defensa}

El sistema de defensa (SD) es un sistema encargado de mediar la sensación subjetiva de miedo y ansiedad, así como las respuestas autónomas (adaptativas) del sistema nervioso simpático como aumento de la frecuencia cardiaca, dilatación de la pupila, aumento de la presión arterial, dilatación de los vasos sanguíneos y la estimulación de las glándulas sudoríparas, que son necesarias para emprender la huida, escapar o luchar ante una situación amenazante de supervivencia (Próspero-García, 2018).

Sin embargo, estas respuestas autónomas también se presentan durante el síndrome de abstinencia (APA, 2013), las principales características de este síndrome son: irritabilidad, enojo, agresividad, nerviosismo o ansiedad, dificultad para dormir, pérdida del apetito y de la masa corporal; inquietud y ánimo depresivo; entre otras.

Algunas estructuras cerebrales que lo componen son, la amígdala principal núcleo procesador de miedo del cerebro (Hariri et al., 2002), que se ha observado sobreexcitado durante el síndrome de abstinencia. El lóbulo de la ínsula que se encarga de la percepción (identificar e interpretar) los estímulos sensoriales provenientes del ambiente interno. Es decir, participa en monitorear el estado corporal (Lamm et al., 2010). Por ejemplo, cuando una persona ingiere un alimento que le produce dolor de estómago y vómito, la ínsula en interacción con amígdala lo registran como un alimento nocivo para el organismo. Estas dos estructuras reciben proyecciones de sistemas encargados de la percepción de los sabores, por ello, algunas drogas como el alcohol y la marihuana que pasan por la lengua, activan el SMR a través del hipotálamo (que regula la sensación de hambre, la saciedad y el balance energético; Prospéro-

Vivat Academia. Revista de Comunicación. 2021, nº 154, 19-40 
García, 2014) y promueven la ingesta porque la ínsula y la amígdala lo reconocen como algo "seguro", es así como las drogas afectan los sistemas cerebrales que utilizamos para consumir los alimentos, favoreciendo la adicción.

Cómo hemos visto estos dos sistemas están involucrados en la generación de la adicción y el abuso de las drogas como cannabis, opiáceos, cocaína, anfetaminas, metanfetaminas y éxtasis, por mencionar los más importantes. Algunos de los daños asociados con la toxicidad de las drogas son problemas cardiovasculares, síntomas psiquiátricos y neurológicos (Buttner, 2011). A largo plazo el consumo de las drogas puede causar en el cerebro, pérdida neuronal, alteraciones neurodegenerativas, daño axonal, cambios en la microvasculatura, entre otros, que en conjunto desencadenan factores tóxicos, vasculares e hipóxicos que interactúan y dan como resultado daños generalizados en el sistema nervioso central. Así mismo, algunas drogas han sido asociadas con el desarrollo de enfermedades degenerativas como el Párkinson.

\section{Los dos sistemas del marketing}

\section{El marketing y el sistema de motivación y recompensa}

Hasta ahora sabemos que el SMR, como el SD son usurpados por las drogas de abuso y las consecuencias que esto tiene, sin embargo, estos dos sistemas tienen la función de crear conductas adaptativas que nos permitan sobrevivir, como se mencionó, el SMR tiene la función de asegurar la repetición de conductas como dormir, comer, beber y procrear; sin embargo, este sistema va más allá de la supervivencia, porque el ser humano a diferencia de los animales es capaz de sentir placer al escuchar música (Shany, et al., 2019) o contemplar una obra de arte (Skov y Nadal, 2020).

Debido a que el SMR no tiene receptores periféricos, el placer no es un estímulo per sé, sino un constructo que el cerebro crea (Próspero-García, 2014), lo que es placentero para unos, no lo es para otros. El principal reto de los estudios de neuromarketing es explicar la actividad cerebral que subyace al comportamiento del consumidor y cómo los estímulos del marketing interfieren en el proceso de decisión de compra. Algunos estudios de neuromarketing han encontrado la activación de los circuitos de recompensa asociados con las marcas favoritas (Reimann y et al., 2012; Schaefer y et al., 2011), la lealtad a las marcas (Plassman, et al., 2007), las recompensas monetarias (Knutson, et al., 2001), los autos deportivos (Britt, 2004), los empaques atractivos (Stoll y et al., 2008) y la asociación precio calidad (Plassman y et al.., 2008). Conocer los mecanismos neurales permitirá a los comerciantes anticipar los deseos de los consumidores y generar mejores estrategias de marketing.

Plassmann y su equipo (2007) encontraron los marcadores neuronales asociados a la lealtad de los consumidores hacia las marcas y las tiendas que las distribuyen. Fueron elegidos 22 participantes asiduos a centros comerciales y los dividieron en dos grupos: el primero, formado por los que asisten con mayor frecuencia al centro comercial y gastan más de 250 euros mensuales; y el segundo, por los que asisten una 
vez al mes y gastan 50 euros o menos. Mostraron que los participantes del primer grupo estaban dispuestos a pagar más por los productos de sus marcas favoritas, en comparación con los del segundo grupo. Lo que se relacionó con la actividad de la corteza prefrontal ventromedial (CPFvm; toma de decisiones emocionales), el núcleo accumbens (NAc; sensación subjetiva de placer) y la corteza del cíngulo anterior (CCA; anticipación a la recompensa o a la detección de conflicto entre dos o más alternativas). Por otra parte, el logotipo de las tiendas generó la activación del NAc, lo que sugiere el agrado por la tienda, pero, no se reportaron resultados significativos en la activación de estas estructuras en los participantes del segundo grupo.

¿Por qué algunos consumidores son leales a las marcas y otros cambian constantemente? Dar respuesta a esta pregunta resulta difícil. Sin embargo, Schaefer y sus colaboradores (2011) encontraron que los participantes que presentan un rasgo de personalidad llamado "neuroticismo" (que es la tendencia a experimentar emociones desagradables con facilidad, como el enojo o la ansiedad) desarrollan con más facilidad lealtad hacia las marcas, y presentan una mayor actividad del NAc ante la presencia de sus marcas favoritas vs. marcas desconocidas. Estos hallazgos posiblemente se deban a que las personas que presentan este rasgo de personalidad suelen tener una baja tolerancia al estrés, por lo que generalmente eligen opciones seguras (Widiger y et al., 2009), que en este caso pudiera influir en la elección de los productos con los que han tenido experiencias positivas.

De igual forma, Reimann (2012) también estudió la actividad cerebral de los consumidores ante sus marcas favoritas, descubrieron una relación entre la activación de la corteza insular, una estructura cerebral que se encarga de censar los estímulos interoceptivos que pueden ser producidos por emociones (p.ej. lo que comúnmente llamamos "mariposas en el estómago"), y el agrado por una marca. Además, encontraron que el interés por una nueva marca disminuye con el tiempo, pero el recuerdo de la experiencia positiva se mantiene.

Uno de los primeros estudios en describir como las recompensas monetarias activa al NAc, fue el realizado por Knutson y su equipo (2001) quienes además demostraron la implicación del núcleo caudado medial (NCm) en la anticipación al castigo o a la recompensa monetaria.

Britt (2004), realizó una investigación para la agencia de autos Chrysler, en la que participaron voluntarios varones a quienes les mostró fotografías de autos deportivos de la marca Sedan y autos pequeños, y tenían que calificar el atractivo de cada uno de ellos. Los autos deportivos obtuvieron mejores calificaciones y una mayor activación del NAc y del giro fusiforme un área cerebral asociada al reconocimiento de rostros, en comparación con los otros autos. Posiblemente los autos deportivos sean un objeto altamente atractivo para los varones y si Chrysler ofreciera autos de bajo costo con algunas características que tienen los autos deportivos, podría llegar a este nicho de mercado que sueña, con tener un auto deportivo. También, el diseño del empaque de un nuevo producto puede ser crucial en su lanzamiento al mercado. El estudio realizado por Stoll y et al., (2008) muestra la preferencia de los consumidores por

Vivat Academia. Revista de Comunicación. 2021, nº 154, 19-40 
empaques atractivos (vs. empaques no atractivos), y la activación de la CPFm, la corteza orbito frontal (COF; jerarquización de las recompensas), la CCA y la corteza del cíngulo posterior (CCP), áreas cerebrales relacionadas con el procesamiento de la recompensa, la toma de decisiones y la memoria afectiva. Los empaques atractivos podrían influir durante la toma de decisiones de compra, sobre todo si se trata de un producto desconocido que se quiere posicionar en el mercado.

Existen diversos elementos que los comerciantes deben cuidar para que su producto sea elegido entre las ofertas disponibles, tales como la marca, los atributos físicos de los productos y la satisfacción de los clientes, no obstante, los precios juegan un papel muy importante en la elección de los productos (Pinkse y et al., 2002), así lo demuestra el estudio de Plassmann (2008) encontró que la modificación del precio de un producto cambia la percepción de agrado de los consumidores. En este estudio participaron 20 voluntarios, a los que se les instruyó para que dentro del resonador degustaran 5 tipos diferentes de vinos "Cabernet Suvignon", y calificaran el agrado o desagrado por los mismos. Lo que los participantes no sabían es que solamente se trataba de tres vinos: el vino uno, fue servido en dos ocasiones, en la primera, al precio de venta real de 5 dólares y en la segunda con un precio ficticio de 45 dólares; de igual forma, el vino dos, fue servido dos veces, con un precio ficticio de 10 y al precio real de 90 dólares. Se utilizó un tercer vino de 35 dólares como control. Los resultados indican que los voluntarios otorgaron mayores calificaciones de agrado por el sabor de los vinos costosos (45 y 90 dólares) en comparación con los vinos baratos. A nivel cerebral se presentó una mayor activación de la COF, CPFvm y de la CCA, al degustar el vino de 90 dólares vs. el vino de 10 dólares. Durante la toma de decisiones la activación de la CCA y de CPFvm ha sido asociada con la expectativa de la recompensa (Etkin y et al., 2015), mientras que la COF juega un papel fundamental en la codificación las experiencias placenteras (Blood y Zatorre, 2001; De Araujo y et al., 2003). Gracias a este estudio es posible conocer que las experiencias placenteras no sólo dependen de las propiedades sensoriales, sino también, de la integración de las expectativas positivas ("es costoso, debe ser muy bueno") que los consumidores tienen al probar los productos. El cerebro evalúa las experiencias placenteras a través del SMR que experimenta con los productos, los servicios o dentro de los centros comerciales, esta información le servirá para tomar futuras decisiones de compra.

Estos estudios nos permiten observar la aplicación que tienen las neurociencias del consumidor en la generación y en la validación de estrategias de marketing, además de poder observar las implicaciones o el impacto que tienen los estímulos en la conducta de los consumidores; este enfoque es compatible con las prácticas que proponemos se desarrollen en los estudios de neuromarketing social.

\section{El marketing y el sistema de defensa}

Se sabe que los rostros angustiados son capaces de aumentar la actividad de la amígdala (derecha; Poljak y Valenta, 2020), principal estructura asociada con el SD; imaginemos que estamos sentados en la terraza de un restaurante y vemos pasar por la calle a algunas personas corriendo, cuyos rostros muestran angustia, piense, cuál

Vivat Academia. Revista de Comunicación. 2021, nº 154, 19-40 

comunidad

sería su reacción, seguramente usted se sentirá inquieto con esa situación porque algo "malo" podría estar pasando; es así como el sistema de defensa actúa para alertarnos de situaciones que pudieran ser peligrosas para nosotros. De igual manera, las estrategias de marketing de ciertas categorías de producto o servicio aprovechan la activación del SD para promover su consumo, ¿Cuál es la causa?

Aunque hasta ahora no existen estudios de neuromarketing que hayan buscado propositivamente la activación de este sistema, algunos estudios de neuroeconomía y de neurociencias, nos ayudan a comprender los marcadores neuronales asociados a los estímulos aversivos del marketing, como veremos a continuación. Capturar la experiencia de "miedo" de los participantes es uno de los principales desafíos de los estudios de neuroimagen; la investigación realizada por Kinreich (2011), mostró la activación de la amígdala dorsal derecha mientras los participantes observaban extractos de películas de terror, lo que correlacionó con la respuesta declarada de los participantes: tengo miedo. De igual manera, otros estudios han encontrado que la activación de la amígdala es mayor cuando se presentan expresiones faciales de enojo y miedo en comparación con escenas de catástrofes (Hariri y et al., 2002).

Después de conocer estos estudios que describe los estímulos aversivos que son capaces de activar el SD, ¿cómo podemos transformar esta información en estrategias de marketing?, las aseguradoras sin conocer las bases neurales del miedo, por años han utilizado en su publicidad escenas de choques, catástrofes naturales, enfermedades, incendios, entre otros estímulos aversivos que, de acuerdo con el IAPS (Xu, et al., 2017), tienen una valencia emocional negativa alta y son capaces de activar el SD. En el transcurso de los anuncios o del discurso de los agentes de seguros, el SD se va activando, generando ansiedad, temor, y otras emociones negativas, sin embargo, la solución y la tranquilidad para que nada de eso pase, la tienen ellos, al comprar sus productos, nada pasará.

\section{Neuromarketing social}

La mercadotecnia social como se describió al inicio, no tiene fines de lucro y su principal interés son las causas sociales y el cambio de conductas en la población, por ello, nosotros queremos proponer el término neuromarketing social, como un diferenciador de las prácticas del neuromarketing lucrativo. A pesar de que en la actualidad existen innumerables estudios de neuromarketing tanto en la práctica comercial como en el ámbito académico, los estudios del neuromarketing social son muy escasos, a continuación, describiremos las pocas investigaciones que hay en la literatura del neuromarketing que se enfocan en causas sociales como la obesidad y las adicciones.

La industria de alimentos ha dirigido agresivas estrategias de marketing a los niños, contribuyendo al problema de la obesidad infantil en todo el mundo. De igual forma, el estudio de Domínguez-Marín (2017) evaluó la toma de decisiones de compra de productos alimenticios en niños, en función de la densidad energética de los alimentos y la promoción de ventas, además de mostrar por primera vez los marcadores 

comunidad

electrofisiológicos o potenciales relacionados con eventos (PRE) asociados a la toma de decisión de compra, que son respuestas cerebrales eléctricas, con alta resolución temporal que pueden asociarse con el procesamiento cognitivo específico de estímulos determinados. Veintiún niños participaron en este estudio, en el que resolvieron dos tareas. En la primera tarea, tenían que decidir si comprasen los productos alimenticios de alta densidad energética (ADE) o de baja densidad energética (BDE), con y sin promoción. En la segunda, clasificaron los productos como saludables (BDE) o no saludables (ADE).

Los resultados de la segunda tarea muestran que los niños discriminaron de manera confiable la información visual de los alimentos de acuerdo con su contenido calórico, identificándolos como saludables y no saludables. Sin embargo, las respuestas conductuales más rápidas fueron para los alimentos saludables que para los alimentos no saludables, este efecto pudiera estar dado porque la ADE tiene señales más importantes que las de la BDE que atraen y mantienen la atención por más tiempo (Junghans, et al., 2015; Meule \& Kübler, 2014). Por otro lado, las respuestas eléctricas cerebrales de los PRE no detectaron diferencia significativa entre alimentos de ADE y alimentos de BDE. Este hallazgo fue inesperado ya que otros estudios realizados con adultos han observaron una mayor amplitud para alimentos de ADE que para alimentos de BDE (Toepel, et al., 2009). Es probable que la falta de diferencias en la actividad eléctrica cerebral entre ADE y BDE en este estudio se diera porque la evaluación fue hecha en población infantil. Además, de manera conductual, la promoción de ventas aumentó la compra de los productos alimenticios, independientemente de su contenido calórico. De igual forma se observó una mayor actividad eléctrica cerebral asociada con la atención selectiva y la toma de decisiones cuando el alimento saludable y no saludable tenía promoción de venta. Lo que quiere decir que no importa la calidad del alimento, el niño va a preferir la promoción de venta. Esta información resulta muy útil, porque de manera científica podemos decirles a las grandes corporaciones que pueden vender alimentos saludables sin perder rentabilidad.

Por otro lado, el estudio de Bruce y et al., (2010) sugiere que las redes neuronales relacionadas con la motivación por los alimentos están activas desde la infancia. La investigación fue realizada con un grupo de niños obesos y lo comparó con otro grupo de niños con parámetros de peso adecuados a la edad, y observaron la actividad cerebral por medio de RMF. Durante el experimento, se les mostraron a los niños logotipos de marcas conocidas de alimento; en dos etapas, privación de alimento y saciedad. Se observó que ambos grupos tuvieron una activación significativa de las regiones límbicas y paralímpicas (regiones asociadas a las emociones) durante el ayuno. Sin embargo, los niños obesos presentaron una hipersensibilidad ante los estímulos alimenticios y las marcas, en comparación con los de peso saludable. La activación de estas zonas no disminuyó en los niños obesos cuando se encontraron en el estado de saciedad. Las regiones cerebrales activadas por los alimentos forman parte del sistema de motivación recompensa que están asociadas a la sensación subjetiva de placer y que tienen un papel fundamental en el procesamiento de las emociones y del control cognitivo. La sensibilidad de los niños obesos ante estos estímulos de la

Vivat Academia. Revista de Comunicación. 2021, nº 154, 19-40 
mercadotecnia lucrativa podría ser una de las causas del aumento de la motivación por consumir alimentos y el hacerlo en exceso. Estos hallazgos neurofisiológicos muestran de una manera objetiva el impacto que las estrategias de la mercadotecnia lucrativa tienen sobre el cerebro de los niños obesos. Por un lado, las investigaciones del neuromarketing social pueden contribuir al estudio del daño que pueden generar las estrategias del marketing lucrativo sobre las poblaciones y, por otro lado, para que los estímulos de las campañas de marketing social sean más efectivos.

Una de las estrategias políticas sanitarias que actualmente están utilizando algunos países para concientizar a la población sobre el daño que pueden tener algunos productos como alimentos y drogas lícitas, es el etiquetado sobre la cantidad de calorías, azúcares y grasas que contienen los alimentos, así como el uso de mensajes que advierten sobre su posible daño (Grabenhorst, et al.., 2013). En el estudio de Grabenhorst y colaboradores, encontraron que la activación de la amígdala es capaz de predecir el cambio en la toma de decisiones por alimentos no saludables, cuando el empaque tiene un etiquetado que advierte sobre los riesgos a la salud que puede causar el consumo del alimento. Y decantar la toma de decisión por un alimento más saludable. Este estudio es el primero en describir los mecanismos del sistema de defensa asociados con las estrategias de marketing social.

Además de las respuestas cerebrales, existen indicadores fisiológicos que pueden funcionar como indicadores de los procesos cognitivos de los participantes y que permiten medir los estímulos del marketing. El eye traking o rastreo ocular, es una técnica que registra los movimientos oculares de los participantes mientras observan imágenes publicitarias o productos. Su análisis permite realizar mapas de calor en los que se identifican las zonas que atraen con mayor frecuencia la mirada y así poder identificar la información que puede ser relevante o irrelevante para los consumidores (Holmqvist, et al., 2011). Apoyados en esta técnica Maynard (2013) midió la atención visual de adolescentes entre 14 y 19 años al observar anuncios de salud en los empaques de cigarros. $Y$ encontraron que los fumadores experimentados y los de fin de semana tienen una mayor atención hacia los anuncios de advertencia en los empaques genéricos comparados con los empaques de marca. Mientras que los que fuman una vez al día (y que tienen poco tiempo de ser fumadores) parecen evitar los anuncios de advertencia de los empaques de cigarros en general. Y los que nunca han fumado ponen mayor atención a las advertencias que aparecen en los empaques con marca o genéricos, lo que puede reflejar su decisión por no fumar. Estos resultados pueden sugerir que tanto los adolescentes no fumadores, como los que llevan tiempo haciéndolo son conscientes de los daños que puede causar el consumo de la nicotina. Sin embargo, aquellos que apenas inician con esta conducta, ignoran las advertencias porque desean continuar haciéndolo. El análisis de la conducta es muy importante para poder generar estrategias de marketing social que funcionen en distintas poblaciones, el problema de las adicciones es un problema que en la mayoría de los casos tiene una comorbilidad con trastornos psicológicos o psiquiátricos (Rowe y et al., 2004), por ello para poder lograr una efectiva prevención se deben conocer los drivers de consumo de los adolescentes por sustancias de abuso. 
Cervantes-Aldana, J. y Domínguez-Marín, M.

Ampliando los horizontes del neuromarketing: disminución de los problemas adictivos en la comunidad

\section{DISCUSIÓN}

Pensamos que la mejor forma donde el neuromarketing social podría empezar a realizar investigaciones es en el campo de las adicciones donde se gastan millones de dólares para dar a conocer campañas preventivas que alejen a los adolescentes de las drogas lícitas e ilícitas (Wakefiel, et al., 2006). En su trabajo, Mercedes García-Toribio (2011) afirma del fracaso de 28 campañas de prevención contra las drogas en España; y concluye que en ninguna de ellas ha dado resultado debido a que las campañas no han sido dirigidas a los adolescentes, sino a la sociedad en general, cuyos mensajes están orientados a la toma de conciencia y no a inducir cambios en la conducta.

Debemos tomar en cuenta, que las neurociencias aplicadas a la creación de campañas sociales nos brindan la posibilidad de validar y elegir los mejores estímulos que serán utilizados con la finalidad de predecir las activaciones cerebrales de los espectadores y con ello la aceptación de la campaña. Sin embargo, las estrategias de segmentación (grupos, horarios y medios) la investigación de la conducta de los adolescentes (usos, hábitos y actitudes) para la creación de mensajes efectivos que logren conectar con ellos, son parte de las prácticas del marketing tradicional.

Por ello sugerimos, en primera instancia, realizar una investigación profunda de los drivers de consumo que llevan a los adolescentes a experimentar con las drogas y a la repetición de la conducta, que pueden ser inherentes a las condiciones sociales y culturales de cada región, con el objetivo de desarrollar mensajes y contenido que conecte con las audiencias. En segundo lugar, llevar a cabo una adecuada segmentación de los públicos a los que se va a dirigir la campaña, como vimos, los adolescentes presentan conductas de riesgo asociadas al desarrollo de la corteza prefrontal (Jadhav y Boutrel, 2019), que los lleva a cometer errores de juicio, además de otros factores de riesgo psicosocial asociados a esta etapa, por lo que son altamente influenciables por los pares para realizar el primer consumo (García-Toribio, 2011). Por ello, los padres y los profesores juegan un papel muy importante en la prevención de las adicciones, quienes también deberían ser parte de la segmentación y dirigir mensajes específicos. Por otro lado, también sugerimos llevar a cabo campañas de educación preventiva e informativa dirigidas a niños y preadolescentes, que se encuentran en una etapa de desarrollo cerebral distinta (Sisk, et al., 2005), lo que les permite comprender desde otra perspectiva la problemática, y no esperar a que sean adolescentes para informarlos sobre los posibles daños del consumo de las drogas de abuso.

Por último, partiendo de un conocimiento profundo de la conducta de los segmentos elegidos y de la problemática de cada región, sugerimos validar a través del conocimiento de las neurociencias y de sus herramientas, los estímulos que serán utilizados en la campaña. A través del conocimiento de los estudios neurocientíficos en general, se sabe que la música (Shany, et al., 2019), las obras de arte (Skov y Nadal, 2020), los rostros hermosos (Aharon y et al., 2001), los olores (Jiang, et al., 2015) y los coros (Altenmüller, et al., 2013), son capaces de activar el SMR, de igual forma, como se describió antes, existen otros estímulos aversivos, como los rostros angustiados

Vivat Academia. Revista de Comunicación. 2021, n 154, 19-40 

comunidad

(Poljak y Valenta, 2020) y las imágenes de catástrofes (Hariri y et al., 2002) que pueden activar el SD, con base en esta información podemos seleccionar estímulos previamente validados por las neurociencias y así crear por ejemplo, un mejor storytelling para un comercial, que lleve al espectador a través de diferentes activaciones cerebrales, que posteriormente podemos corroborar a través de las herramientas neurocientíficas. Por último, proponemos, realizar estudios experimentales (casos control y experimental) en los que se midan los efectos en el cambio de conductas y actitudes de los adolescentes, asociados a las campañas de prevención.

\section{CONCLUSIONES}

Gran parte de la literatura del marketing y del neuromarketing se ha dedicado al área comercial. Sin embargo, el surgimiento del marketing social (Kotler y Levy, 1971) le dio un nuevo sentido al uso de las herramientas del marketing, donde muchos académicos enfocaron sus esfuerzos para apoyar causas sociales. Como mostramos, existen estudios de neuromarketing que contribuyen de manera significativa al quehacer del marketing lucrativo, por ello proponemos utilizar las herramientas neurocientíficas para desarrollar estudios con problemáticas sociales. Aunque encontramos pocos estudios que persiguen este fin, no se le da el calificativo de neuromarketing social, ni existe en la literatura ningún término que lo haya utilizado de esta forma.

Por otro lado, el problema de la drogadicción es una cuestión muy grave para la sociedad por sus consecuencias sociales, psicológicas y económicas. Si bien es muy complicado tratar al adicto para que deje de consumidor drogas, pensamos como muchos especialistas en prevención de adicciones, que lo mejor es prevenir que los jóvenes adolescentes en sus etapas más tempranas, antes de que se vuelvan adictos, en lugar de sacarlos del sufrimiento para ellos y sus familiares del daño que causan en el cuerpo humano las drogas.

También encontramos en la revisión de la literatura que las campañas preventivas no han dado los resultados esperados. Es un problema monumental que requiere no solo el concurso de investigadores académicos en varias áreas sino la participación de la familia, la sociedad en general y el Estado. Las campañas no han movido suficientemente a la acción para alejarlos de las drogas. No hemos encontrado que en esas campañas se hubieran utilizados herramientas del neuromarketing. Finalmente, creemos que es importante la creación de esta nueva área de investigación que identifique y separe los tipos de investigación en neuromarketing lucrativo de aquellos enfocados a resolver problemáticas sociales. Al hacer esto, al etiquetar de esta forma el neuromarketing social, permitirá que, en el futuro más académicos, alumnos universitarios e investigadores de institutos, dirijan sus esfuerzos a proyectos con sentido social como en propuesto en esta ponencia sobre la prevención de adicciones. 
Cervantes-Aldana, J. y Domínguez-Marín, M.

Ampliando los horizontes del neuromarketing: disminución de los problemas adictivos en la comunidad

\section{REFERENCIAS}

Aharon, I., Etcoff, N., Ariely, D., Chabris, C. F., O'connor, E., \& Breiter, H. C. (2001). Beautiful faces have variable reward value: fMRI and behavioral evidence. Neuron, 32(3), 537-551. https://doi.org/10.1016/S0896-6273(01)00491-3

Altenmüller, E., Kopiez, R., \& Grewe, O. (2013). Strong emotions in music: are they an evolutionary adaptation?. In Sound-Perception-Performance. 131-156. Springer, Heidelberg. https://doi.org/10.1007/978-3-319-00107-4_5

Andreasen, A. R. (1994). “Social marketing: its definition and domain”. Journal of Public Policy \& Marketing, 13(1), 108-114. https:// doi.org/10.1177/074391569401300109

APA (2013). Manual diagnóstico y estadístico de los trastornos mentales, American Psychiatric Association. American Psychiatric Association (5. thed.[DSM-5]).

Aron, A., Fisher, H., Mashek, D. J., Strong, G., Li, H., \& Brown, L. L. (2005). Reward, motivation, and emotion systems associated with early-stage intense romantic love. Journal of neurophysiology, 94(1), 327-337. https:// doi.org/10.1152/jn.00838.2004

Blood, A. J., \& Zatorre, R. J. (2001). Intensely pleasurable responses to music correlate with activity in brain regions implicated in reward and emotion. Proceedings of the National Academy of Sciences, 98(20), 11818-11823. https://doi.org/10.1073/pnas.191355898

Bruce et al., (2013). Brain responses to food logos in obese and healthy weight children. The Journal of pediatrics, 162(4), 759-764 https://doi.org/10.1016/j.jpeds.2012.10.003

Burrone et al., (2010). Análisis de la frecuencia de experimentación y consumo de drogas de alumnos de escuelas de nivel medio. Revista Latino-Americana de Enfermagem, 18(SPE), 648-654 https:/ / doi.org/10.1590/S0104-11692010000700023

Büttner, A. (2011). The neuropathology of drug abuse. Neuropathology and applied neurobiology, 37(2), 118-134 https://doi.org/10.1111/j.1365-2990.2010.01131.x

Cervantes-Aldana, F. (1980). The application of social marketing in the prevention of diarrheal diseases: the case of Mexico. Ph.D. Thesis. The University of Texas at Austin.

De Araujo, I. E., Kringelbach, M. L., Rolls, E. T., \& McGlone, F. (2003). Human cortical responses to water in the mouth, and the effects of thirst. Journal of neurophysiology, 90(3), 18651876 https://doi.org/10.1152/jn.00297.2003

Domínguez-Marín (2017). La mercadotecnia social, el consumo de alimentos no saludables y la función del cerebro en niños de 11 y 12 años. Tesis Doctoral, mayo, 2017, División de 
Cervantes-Aldana, J. y Domínguez-Marín, M.

Ampliando los horizontes del neuromarketing: disminución de los problemas adictivos en la comunidad

Estudios de Posgrado, FCA, UNAM, México. http://132.248.9.195/ptd2017/mayo/0758988/0758988.pdf

Etkin, A., Egner, T., \& Kalisch, R. (2011). Emotional processing in anterior cingulate and medial prefrontal cortex. Trends in cognitive sciences, 15(2), 85-93 https:// doi.org/10.1016/j.tics.2010.11.004

Fadaei, M. H., Farokhzadian, J., Miri, S., \& Goojani, R. (2020). Promoting drug abuse preventive behaviors in adolescent students based on the health belief model. International journal of adolescent medicine and health, 1(ahead-of-print) https://doi.org/10.1515/ijamh-2019-0170

Fischer de la Vega, L., \& Espejo Callado, J. (2004). Mercadotecnia (3a ed. ed.). México: McGraw-Hill.

García-Toribio (2011), Estrategias de comunicación basadas en las conductas y el ocio de los jóvenes y orientadas a la prevención de la drogadicción. Tésis Doctoral, Complutense de Madrid, España.

Grier, S., Bryant, C. (2005), "Social marketing in public health", Annual Review Public Health, Vol. 319-339 https://doi.org/10.1146/annurev.publhealth.26.021304.144610

Gómez, J., \& Muñoz, M. (2000). Factores de riesgo relacionados con la influencia del grupo de iguales para el consumo de drogas en adolescentes. Psicología conductual, 8, 19-32.

Grabenhorst, F., Schulte, F. P., Maderwald, S., \& Brand, M. (2013). Food labels promote healthy choices by a decision bias in the amygdala. Neuroimage, 74, 152-163. https://doi.org/10.1016/j.neuroimage.2013.02.012

Hariri, A. R., Tessitore, A., Mattay, V. S., Fera, F., \& Weinberger, D. R. (2002). The amygdala response to emotional stimuli: a comparison of faces and scenes. Neuroimage, 17(1), 317-323 https://doi.org/10.1006/nimg.2002.1179

Hillenbrand, P., \& Cervantes, J. (2015). Neuromarketing: una nueva forma de entender la mente del consumidor (Cuarta reimpresión ed.). Publicaciones Empresariales UNAM.FCA Publishing https://doi.org/10.18004/ucsa/2409_ $\underline{8752 / 2018.005(02) 036-044}$

Holmqvist, K., Nyström, M., Andersson, R., Dewhurst, R., Jarodzka, H., \& Van de Weijer, J. (2011). Eye tracking: A comprehensive guide to methods and measures. OUP Oxford http:// ukcatalogue.oup.com/product/9780199697083.do

Jadhav, K. S., \& Boutrel, B. (2019). Prefrontal cortex development and emergence of self-regulatory competence: the two cardinal features of adolescence disrupted in

Vivat Academia. Revista de Comunicación. 2021, nº 154, 19-40 
Cervantes-Aldana, J. y Domínguez-Marín, M.

Ampliando los horizontes del neuromarketing: disminución de los problemas adictivos en la comunidad

context of alcohol abuse. European Journal of Neuroscience, 50(3), 2274-2281 https://doi.org/10.1111/ejn.14316

Jiang, T., Soussignan, R., Schaal, B., \& Royet, J. P. (2015). Reward for food odors: an fMRI study of liking and wanting as a function of metabolic state and BMI. Social $\begin{array}{llll}\text { cognitive affective neuroscience, 10(4), 561-568 } & \end{array}$ https://doi.org/10.1093/scan/nsu086

Junghans, A. F., Hooge, I. T. C., Maas, J., Evers, C., \& De Ridder, D. T. D. (2015). Children and adults visual attention to healthy and unhealthy food. Eating Behaviors, 17, 90-93 https:// dx.doi.org/10.1016/j.eatbeh.2015.01.009

Kerin, R., Hartley, S., \& Rudelius, W. (2009). Marketing (9a ed. ed.). China: McGrawHill.

Kinreich, S., Intrator, N., \& Hendler, T. (2011). Functional cliques in the amygdala and related brain networks driven by fear assessment acquired during movie viewing. Brain connectivity, 1(6), 484-495 https://doi.org/10.1089/brain.2011.0061

Knutson, B., Adams, C. M., Fong, G. W., Hommer, D. (2001). Anticipation of Increasing Monetary Reward Selectively Recruits Nucleus Accumbens. The Journal of Neuroscience, (21)16, 159 https:/ / doi.org/10.1523/jneurosci.21-16-j0002.2001

Kotler, P., \& Lee, N. R. (2007). Social marketing: Influencing behaviors for good. Thousand Oaks, CA: Sage.

Kotler, P., \& Gerald, Z. (1971). Social marketing: an Approach to planned change. Journal of marketing, 35:5 https://doi.org/10.1177/002224297103500302

Kotler, P., \& Roberto, E. (1992). Mercadotecnia social (1ra ed.). Ed. Diana

Kotler, P.and Sidney Levy (1969), "Broadening the Concept of Marketing," Journal of Marketing. 33, 10-15 https://doi.org/10.2307/1248740

Lamm, C., \& Singer, T. (2010). The role of anterior insular cortex in social emotions. Brain Structure and Function, 214(5-6), 579-591 https:// doi.org/10.1007/s00429-010$\underline{0251-3}$

Lobato-Rincón, L. L. (2015). Pupilometría dinámica mesópica: valores patrón y su variación con el efecto del alcohol. https:/ / eprints.ucm.es/id/eprint/32810/1/T36294.pdf

Martin-Armario. E. (1997) Mercadotecnia Madrid. Ed. Ariel.

Martínez Martínez, K. I., Salazar Garza, M. L., Pedroza Cabrera, F. J., Ruiz Torres, G. M., y Ayala Velázquez, H. E. (2008). Resultados preliminares del programa de 
Cervantes-Aldana, J. y Domínguez-Marín, M.

Ampliando los horizontes del neuromarketing: disminución de los problemas adictivos en la comunidad

intervención breve para adolescentes que inician el consumo de alcohol y otras drogas. Salud mental, 31(2), 119-127.

Maynard, O. M., Munafò, M. R., \& Leonards, U. (2013). Visual attention to health warnings on plain tobacco packaging in adolescent smokers and non-smokers. Addiction, 108(2), 413-419 https://doi.org/10.1111/j.1360-0443.2012.04028.x

McCarthy, J., \& Perreeault, W. (1997). Marketing (11a ed. ed.). McGraw-Hill.

Méndez-Díaz, M., Torres, B. M. R., Morelos, J. C., Ruíz-Contreras, A. E., y ProspéroGarcía, O. (2017). Neurobiología de las adicciones. Revista de la Facultad de Medicina UNAM, 60(1), 6-16.

Mercado, S. (1998). Mercadotecnia programada (2a ed. ed.). Limusa.

Meule, A., \& Kübler, A. (2014). Double trouble. Trait food craving and impulsivity interactively predict food-cue affected behavioral inhibition. Appetite, 79(04), 174182. https://doi.org/10.1016/j.appet.2014.04.014

Münich Galindo, L., y García Martínez, J. (1990). Fundamentos de administración (5a reimpresión ed.). Trillas.

OMS (2004). Neurociencia del consumo y dependencia de sustancias psicoactivas: resumen. Organización Mundial de la Salud. https://apps.who.int/iris/bitstream/handle/10665/42865/924359124X.pdf

Pinkse, J., Slade, M. E., \& Brett, C. (2002). Spatial price competition: a semiparametric approach. Econometrica, 70(3), 1111-1153 https:// doi.org/10.1111/1468-0262.00320

Plassmann, H., Kenning, P., Ahlert, D. (2007). Why Companies Should Make Their Customers Happy: The Neural Correlates of Customer Loyalty. Advances in Consumer Research, 34, 735-739

Plassmann, H., O’Doherty, J., Shiv, B., Rangel, A. (2008). “Marketing actions can modulate neural representations of experienced pleasantness". PNAS, (105):3. 150154 https://doi.org/10.1073/pnas.0706929105

Poljak Lukek, S., \& Valenta, T. (2020). Neurobiological and Relational Bases for Understanding Aggressiveness. The Person and the Challenges. The Journal of Theology, Education, Canon Law and Social Studies Inspired by Pope John Paul II, 10(1), 155-176 http://dx.doi.org/10.15633/pch.3615

Prospéro-García, O. (2014). El cerebro ADICTO. Ciencia-Academia Mexicana de Ciencias. 65(1), 26-31. 
Cervantes-Aldana, J. y Domínguez-Marín, M.

Ampliando los horizontes del neuromarketing: disminución de los problemas adictivos en la comunidad

Prospéro-García, O. (2018). Las adicciones. UNAM, Dirección General de Divulgación de la Ciencia. Ciudad Universitaria.

Reimann, M., Castaño, R., Zaichkowsky, J., Bechara, A., (2012). How we relate to brands: Psychological and neurophysiological insights into consumer-brand relationships. Journal of Consumer Psychology (22), 128-142 https://doi.org/10.1016/j.jcps.2011.11.003

Reséndiz-Escobar, E., Bustos Gamiño, M. N., Mujica Salazar, R., Soto Hernández, I. S., Cañas Martínez, V., Fleiz Bautista, C.;Villatoro Velázquez, J. A. (2018). Tendencias nacionales del consumo de alcohol en México: resultados de la Encuesta Nacional de Consumo de Drogas, Alcohol y Tabaco 2016-2017. Salud mental, 41(1), 7-15 https://doi.org/10.17711/SM.0185-3325.2018.003

Rowe, C. L., Liddle, H. A., Greenbaum, P. E., \& Henderson, C. E. (2004). Impact of psychiatric comorbidity on treatment of adolescent drug abusers. Journal of Substance Abuse Treatment, 26(2), 129-140 https://doi.org/10.1016/S0740$\underline{5472(03) 00166-1}$

Schaefer, M., Knuth, M., Rumpel, F. (2011). Striatal response to favorite brands as a function of neuroticism and extraversion. Brainresearch, (1425), 83-89 https://doi.org/10.1016/j.brainres.2011.09.055

Shany, O., Singer, N., Gold, B. P., Jacoby, N., Tarrasch, R., Hendler, T., \& Granot, R. (2019). Surprise-related activation in the nucleus accumbens interacts with musicinduced pleasantness. Social cognitive and affective neuroscience, 14(4), 459-470 https://doi.org/10.1093/scan/nsz019

Sisk, C. L., \& Zehr, J. L. (2005). Pubertal hormones organize the adolescent brain and

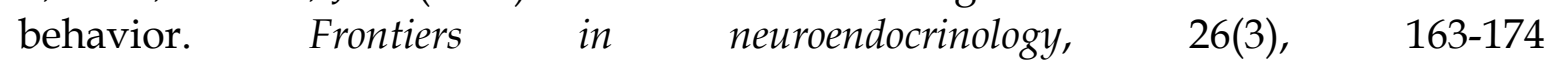
https://doi.org/10.1016/j.yfrne.2005.10.003

Skov, M., \& Nadal, M. (2020). A farewell to art: Aesthetics as a topic in psychology and neuroscience. Perspectives on Psychological Science, 15(3), 630-642 $\underline{\text { https://doi.org/10.1177/1745691619897963 }}$

Stanton, W. (2000). Fundamentos del marketing (11a edición ed.). México: McGraw-Hill.

Stoll, M., Baecke, S., \& Kenning, P. (2008). What they see is what they get? An fMRIstudy on neural correlates of attractive packaging. Journal of Consumer Behaviour, 7(4-5), 342-359 https://doi.org/10.1002/cb.256

Toepel, U., Knebel, J. F., Hudry, J., Coutre, J., Murray, M. M. (2009) The brain tracks the energetic value in food images. NeuroImage (44), 967-974 https://doi.org/10.1016/j.neuroimage.2008.10.005 
Cervantes-Aldana, J. y Domínguez-Marín, M.

Ampliando los horizontes del neuromarketing: disminución de los problemas adictivos en la comunidad

UNODC (2020), Informe Mundial sobre las drogas 2020 de la UNODC: el consumo global aumenta a pesar de que el COVID-19 tiene un impacto de gran alcance en los mercados mundiales de drogas. Oficina de las Naciones Unidas Contra las Drogas y el Delito. https://n9.cl/iifo

Young, S. E., Corley, R. P., Stallings, M. C., Rhee, S. H., Crowley, T. J., \& Hewitt, J. K. (2002). Substance use, abuse and dependence in adolescence: prevalence, symptom profiles and correlates. Drug and alcohol dependence, 68(3), 309-322 https://doi.org/10.1016/s0376-8716(02)00225-9

Wakefield, M., Terry-McElrath, Y., Emery, S., Saffer, H., Chaloupka, F. J., Szczypka, G., Johnston, L. D. (2006). Effect of televised, tobacco company-funded smoking prevention advertising on youth smoking-related beliefs, intentions, and behavior. American Journal of Public Health, 96(12), 2154-2160 https://dx.doi.org/10.2105/AJPH.2005.083352

Xu, Z., Zhu, R., Shen, C., Zhang, B., Gao, Q., Xu, Y., \& Wang, W. (2017). Selecting pureemotion materials from the International Affective Picture System (IAPS) by Chinese university students: A study based on intensity-ratings only. Heliyon, 3(8), e00389 https:/ / doi.org/10.1016/j.heliyon.2017.e00389

\section{AUTORES:}

\section{F. Javier Cervantes Aldana}

Doctor en Mercadotecnia por la Universidad de Texas en Austin; también tiene el grado de Maestro Licenciado en Administración otorgado por la Universidad Nacional Autónoma de México (UNAM). Ha sido catedrático en numerosas Universidades tanto de México como del extranjero. Actualmente es Profesorinvestigador en la División de Investigación de la Facultad de Contaduría y Administración de la UNAM. Es Socio Fundador y Presidente del Consejo de Administración de Psyma Latina, empresa de estudios de mercado que dirigió por más de 10 años y que forma parte del Grupo Psyma AG ,con sede en Alemania y oficinas en 12 países. Es autor de artículos de investigación en diversas publicaciones como el American Marketing Association, Market Research Association, Academy of Marketing Science, entre otros. Es coautor del libro "Neuromarketing: una nueva forma de entender la mente del consumidor.

\section{Miryam M. Domínguez Marín}

Doctora en Ciencias de la Administración por la Universidad Nacional Autónoma de México (UNAM); Realizó el doctorado con el grupo de neurociencias del Dr. Óscar Prospéro-García de la Facultad de Medicina de la UNAM. Y realizó un posdoctorado en el laboratorio de Neurogenómica congnitiva; su línea de investigación es el Neuromarketing Social. Ha participado como ponente en congresos internacionales de neurociencias y de marketing. Actualmente es profesora de Posgrado y de la Facultad de Contaduría y Administración de la UNAM. Además de contar con experiencia 
Cervantes-Aldana, J. y Domínguez-Marín, M.

Ampliando los horizontes del neuromarketing: disminución de los problemas adictivos en la comunidad

práctica en agencias de inteligencia de mercados. Actualmente trabaja en Applied Cognitive Diagnostics, donde desarrolla investigaciones de distintas áreas. 